\title{
Mehrdad Vahabi, The Political Economy of Destructive
}

Power

Cheltenham, Edward Elgar, 2004, 280 pages

Louis Baslé

\section{OpenEdition}

\section{Journals}

Édition électronique

URL : http://journals.openedition.org/ei/1135

DOI : 10.4000/ei. 1135

ISSN : 2553-1891

\section{Éditeur}

Association Économie et Institutions

Édition imprimée

Date de publication : 1 juin 2006

Pagination : 141-151

ISSN : 1775-2329

Référence électronique

Louis Baslé, "Mehrdad Vahabi, The Political Economy of Destructive Power », Économie et institutions [En ligne], 8 | 2006, mis en ligne le 31 janvier 2013, consulté le 24 septembre 2020. URL : http:// journals.openedition.org/ei/1135; DOI : https://doi.org/10.4000/ei.1135 
Compte-rendu de l'ouvrage de Mehrdad Vahabi, The

Political Economy of Destructive Power, Edward Elgar, Cheltenham 2004, 280 pages.

\section{Louis BASLE (CRIISEA, Université de Picardie)}

Bonne idée que de consacrer un ouvrage entier à cet envers, cette part maudite de l'économie politique, la destruction et le pouvoir de destruction. Certes, l'abondance de la bibliographie et des références où figurent majoritairement mais non exclusivement de nombreux économistes, les modèles économiques analysés, disséqués, critiqués, tout cela montre que Mehrdad VAHABI n'est pas le seul économiste qui se soit penché sur la question. Mais, du moins à ma connaissance, notre auteur est le premier qui y ait consacré un ouvrage entier.

Après un résumé succinct, je soulèverai certains points problématiques pour souligner l'intérêt, la pertinence du propos, mais aussi, de l'humble point de vue d'un lecteur critique, ses limites.

L'ouvrage se présente en $\mathbf{5}$ chapitres, le $\mathbf{1}^{\text {er }}$ tenant lieu d'introduction où est défini le pouvoir destructif, en opposition et comparaison avec le pouvoir constructif et le pouvoir moral. Sont ensuite successivement traitées la signification (ch.2), la nature sociale (ch.3), la valeur (ch.4), et enfin les sources du pouvoir destructif. (ch.5).

Le chapitre introductif fera l'objet d'un compte rendu assez détaillé car il pose la problématique d'ensemble. M.VAHABI y propose sa propre définition du pouvoir en perspective avec celles de grands auteurs aussi divers que Hannah ARENDT, Herbert Simon, Kenneth BOULDING et Max WEBER. Il marque ainsi son opposition à ce dernier en estimant qu'il suffit d'une simple potentialité crédible d'influencer les décisions pour qu'il y ait pouvoir. Cela fait, les trois types de pouvoir sont définis. Le pouvoir constructif correspond à la capacité de créer de la valeur (ce qui introduit une équivalence problématique entre production économique et construction), le pouvoir destructif à celle d'en détruire, et enfin le pouvoir moral à celle de former et influencer les opinions et croyances. Les trois pouvoirs sont à la fois séparés l'un de l'autre, opposés l'un à l'autre et encastrés l'un dans l'autre, en ce sens que chacun contient une part des deux autres et, réciproquement, fait partie des deux autres, comme le montrent les figures des pp. 19, 26, 34 et 38.

La séparation des trois pouvoirs, typique du développement moderne, est déjà illustrée dans la célèbre tripartition fonctionnelle de DumÉzIL, tripartition "plus idéale que réelle " (M.VAHABI, p.11) mais analytiquement utile. M.VAHABI esquisse la longue évolution historique vers cette séparation: le pouvoir moral prend d'abord, 
pour s'autonomiser, la forme du pouvoir religieux, bien que longtemps confondu, allié ou inféodé au pouvoir politique, forme visible et, elle aussi, de plus en plus autonome, de pouvoir destructif. Quant au pouvoir constructif, il est d'abord sous la coupe du pouvoir destructif et c'est le développement de la société moderne, en particulier l'avènement du capitalisme, qui développe la séparation. L'auteur pense que la tentative soviétique de revenir sur cette séparation ne sera pas renouvelée.

On l'a vu, par-delà leur séparation, les 3 pouvoirs s'encastrent partiellement les uns dans les autres. Ainsi, le pouvoir destructif inclut les 2 autres. En effet, destruction et construction sont mêlées puisque la destruction est le moment transitoire nécessaire de la production (SCHUMPETER) et que tout apprentissage inclut la destruction partielle des savoirs acquis. Même dans le cas de la destruction pour elle-même (la "négation abstraite" de HEGEL), pouvoir constructif n'est pas absent. Les capacités utilisées par le pouvoir destructif ont des effets externes positifs : ainsi, l'activité destructrice renforce la cohésion et la solidarité collectives M.VAHABI fait ici référence au concept arabe d'assabiya, longuement développé par IBN KHALDUN - en même temps qu'elle nécessite de l'organisation et crée des emplois. Il est vrai que tout cela est contrebalancé par un coût social considérable qui pèse sur les Etats et les sociétés. Le pouvoir destructif mobilise des ressources et implique protection et défense contre le pouvoir destructif de l'ennemi potentiel ou réel. Il est à noter que le développement de la fiscalité et des finances publiques est fortement lié à tout cela. Le pouvoir destructif inclut aussi le pouvoir moral dans la mesure où la destruction mobilise des dimensions morales tant négatives que positives comme le plaisir sadique et la cruauté, le courage et la fraternité.

Ce qui est vrai du pouvoir destructif l'est aussi pour le pouvoir constructif. En particulier, sous le mode capitaliste, la production inclut des processus impersonnels de destruction et d'élimination comme les disparitions d'entreprises et d'emplois, l'exclusion des non propriétaires de moyens de production et, plus généralement, le conflit entre classes sociales. Ces processus inintentionnels se doublent de modes personnalisés et intentionnels : ainsi dans les conflits sociaux qui peuvent prendre des formes plus ou moins violentes avec, le plus souvent, une position asymétrique favorable aux employeurs. A ces conflits peuvent s'ajouter des conflits internes aux classes et groupes. En cas de pouvoir de monopole, la concurrence devient intentionnellement destructrice. Le pouvoir politique peut venir renforcer cette domination. Les périodes de révolution et de transition ont des effets destructeurs intentionnels et non intentionnels du fait du chaos et du vide politique avec, au 
minimum, une "récession transformationnelle" (KORNAI) et donc une destruction de valeur.

Le pouvoir moral est également contenu dans le pouvoir constructif. M.VAHABI fait ici référence à la thèse bien connue de Max Weber sur l'éthique protestante. Les relations économiques aussi sont empreintes de dimensions morales sur ce qui est loyal ou non et l'on débat depuis Adam Smith à propos des relations entre efficacité et justice et des effets moraux positifs ou négatifs de l'économie de marché.

Pour en venir enfin aux aspects destructifs et constructifs du pouvoir moral, on sait la capacité des pouvoirs religieux, idéologique et médiatique à mobiliser et convaincre tant pour détruire (croisades, guerres de religions, campagnes de haine et propagande belliciste) que pour construire. En outre, certaines valeurs éthiques telle que la responsabilité et la liberté individuelles, l'esprit d'entreprise, etc. sont corrélés au développement du pouvoir constructif, en particulier, capitaliste.

Une fois situé le pouvoir destructif, il convient de préciser sa signification et ses dimensions et c'est l'objet du chapitre 2. Pour ce faire, il faut situer le pouvoir destructif par rapport à la théorie morale, à l'intentionnalité et à l'autodestruction. L'auteur nous convie ensuite à une revue des modèles économiques et politicoéconomiques qui traitent de la question. Sur la théorie morale, M.VAHABI conclut à la neutralité morale a priori du pouvoir destructif. Ensuite, il distingue le pouvoir destructif exogène, imputable à la nature, du pouvoir destructif endogène où la volonté humaine est décisive. La destruction endogène est souvent, selon M.VAHABI lequel invoque des auteurs aussi divers et dissemblables que MARX, FREUD et SARTRE - la seule source de changement historique social radical. Ce pouvoir destructif endogène peut aller jusqu'à falsifier l'histoire. La destruction exogène comprend aussi les aspects physiologiques, psychologiques et mentaux de l'individu humain. La frontière qui sépare l'exogène de l'endogène est parfois difficile à tracer par exemple dans le cas de la concurrence. M.VAHABI évoque enfin les comportements d'autodestruction comme l'anorexie, certaines formes de déviance et de criminalité, la radicalité avec risque extrême dont les attentats-suicides, le suicide: l'auteur rattache ces comportements à des facteurs sociétaux. Sur ce point, il souligne la position asymétrique des Afro-américains pris dans des contraintes socio-institutionnelles qui ne leur laisse d'autre choix que la destruction et l'autodestruction.

M.VAHABI passe ensuite à l'analyse de deux grandes familles de modèles: les modèles économiques du conflit et les modèles prenant pour objet la violence politique. L'ensemble de ces modèles souffrent presque tous, selon l'auteur, de la référence à la rationalité 
optimisatrice. Il en résulte que violence et destruction sont toujours ramenées à une étiologie et une raison économiques à des relations coûts-avantages entre activités de prédation ou de production, et que le facteur proprement politique est une fois de plus occulté. Il en résulte en conséquence que toutes les formes de violence et de destruction sont confondues sous le concept de conflit, expression d'une situation sous-optimale. Les réflexions d'HIRSCHMAN sont plus intéressantes en ce qu'elles ne font pas appel à la rationalité optimisatrice. Cependant, l'opinion de M.VAHABI est qu'elles partent de concepts "brouillés": en effet, exit et voice n'ont de significations claires que si l'on sait s'ils expriment ou non une attitude de refus ou d'acceptation des règles dominantes. C'est, pour notre auteur, le déterminant de la question. Or HIRSCHMAN s'inscrit explicitement dans l'hypothèse d'acquiescement à ces règles c'est-à-dire admet que leur refus traduit un dysfonctionnement, une crise. En résumé, M.VAHABI refuse que l'on traite le conflit, et en particulier ses formes violentes, comme l'expression d'un déséquilibre, d'une crise, bref d'une situation anormale. La formation et la fondation de nouvelles règles, bref la constitution, l'institution d'un ordre social - y compris la distinction privé-public - tout cela ne peut se faire sans violence. C'est là, selon M.VAHABI, que réside la nature sociale du pouvoir destructif.

Le chapitre 3 est précisément consacré à cette nature sociale. L'auteur ici formule sa thèse centrale qu'on peut résumer ainsi : le pouvoir destructif, toutes formes de violence comprises, ne constitue pas une aberration, n'est pas " un symptôme de crise, de déséquilibre, d'irrationalité, de déviance ". Au contraire, c'est "une force majeure d'intégration sociale " car il a un rôle essentiel "dans la construction d'un ordre social, l'application des règles, leur changement et le développement de la communication ". Il ne faut donc pas disjoindre pouvoir destructif et ordre social. Toute règle, légale ou illégale, "dérive d'une relation de pouvoir ou de l'usage de la force physique " puisque le pouvoir destructif est à l'origine des règles. M.VAHABI distingue, de ce point de vue, trois types de pouvoir destructif : le pouvoir légal dominant (à ses deux niveaux, statonational et international) qui se réclame des règles en vigueur, le pouvoir révolutionnaire ou anti-système qui veut changer les règles et enfin le pouvoir criminel qui ne respecte que ses propres règles. Dans la continuité de son raisonnement, M.VAHABI montre aussi comment la guerre et son organisation sociale, l'armée sont des creusets d'identification collective, de socialisation et d'intégration de peuples et groupes sociaux a priori étrangers les uns aux autres. A propos de l'intégration, l'auteur, sur la base d'une distinction fondamentale entre les deux grandes fonctions du pouvoir destructif, appropriation et production de règles, opère une discrimination entre 
"les empires de domination", à intégration simplement "extensive " et "les empires territoriaux", à intégration "intensive " Enfin, qu'il pratique la menace, qu'il use de violence, qu'il prenne enfin la forme de la protestation organisée, le pouvoir destructif est une forme parmi d'autres d'expression individuelle mais surtout collective, ce qui nous renvoie à la communication. Le P.D. se ramène finalement à "la préférence pour la violence" en particulier pour les groupes dominant pouvoir destructif s qui opèrent un calcul "coûts-avantages" à propos du maintien de l'ordre social désiré. On aura deviné depuis un moment que l'adversaire est ici le paradigme économique néoclassique. La critique de M.VAHABI s'approfondit avec la question de distinction entre bien collectif (en anglais "public") et bien privé. L'auteur souligne que ce n'est pas la nature des biens qui est ici discriminante mais bien la souveraineté à l'origine des règles et statuts : ainsi, ce n'est pas tant la possibilité ou non des effets d'exclusion et de rivalité qui fait la distinction ; c'est bien plutôt, par exemple, la possibilité ou non de la servitude pour dette. En ce cas, le respect du contrat peut réduire le débiteur à l'état de péon au service de son créancier. En résumé, le pouvoir destructif est à l'origine de tout ordre social, de l'application des règles liées à cet ordre, du changement éventuel des règles et de la forme que prend ce changement ; en toute logique, le pouvoir destructif joue donc un rôle majeur dans la communication sous ses trois dimensions : formation des identifications collectives, processus d'intégration et forme d'expression; enfin, le pouvoir destructif, étant à l'origine de la souveraineté et des règles, fonde les distinctions prétendument naturelles, comme celle entre bien collectif et bien privé.

Avec le chapitre 4, l'auteur aborde la question de la valeur $\mathrm{du}$ pouvoir destructif. Tout d'abord, pour en saisir l'enjeu, il faut se reporter à la réflexion de T.C.SCHELLING placée par M.VAHABI en exergue du ch.I : cette réflexion explique la capacité de nuisance potentielle d'un quelconque "healthy high-shooled graduate" qui pourrait détruire cent fois plus de valeur qu'il n'est susceptible d'en produire. On peut aussi, avec M.VAHABI, évoquer la manière peu orthodoxe dont le clochard et l'enfant du film de CHAPLin The Kid s'y prennent pour gagner leur vie. Où l'on voit la spécificité du pouvoir destructif: ce pouvoir n'est pas le contraire équivalent du pouvoir constructif. En effet, "le pouvoir destructif est plus destructif que le pouvoir constructif n'est créatif, car le processus de destruction de valeur est plus rapide que le processus de création de valeur ". Où l'on voit en conséquence que le pouvoir destructif peut être, si l'on ose dire, immédiatement très productif pour qui sait en user. Ainsi Robinson Crusoe "crée" plus de valeur pour lui-même par son pouvoir destructif dès qu'il dispose d'un ou plusieurs compagnons à exploiter. Cela admis, il ne faut pas en rester à une seule des deux 
fonctions de ce pouvoir, à savoir la fonction appropriative. L'autre fonction, à savoir la fonction de production de règles, est peut-être plus productive à terme. Ces deux fonctions ne doivent pas être séparées de manière étanche, en particulier quand violence ou menace et prédation instituent une nouvelle souveraineté, de nouvelles relations asymétriques légitimées comme celles qui lient Robinson Crusoe et Vendredi.

La fonction appropriative se fonde sur le fait qu'on peut mobiliser des ressources "non seulement pour la production mais aussi pour des objectifs d'appropriation par le vol, le pillage, la guerre, l'insurrection ou autres formes d'activité destructive ". On peut donc opérer un choix entre produire ou "s'approprier les biens produits par d'autres" (PARETO). Ici s'applique un "principe de symétrie et d'équivalence des préjudices " qui se concrétise dans la mise en jeu de sa propre vie contre celles des autres, contre des "droits" d'appropriation de biens voire de personnes. Mais il y a une limite à cette fonction appropriative, limite mise en relief par les modèles marginalistes du conflit : la valeur récupérée par le prédateur est toujours inférieure à la valeur perdue par la proie, ce qui, à terme, implique l'épuisement des ressources appropriables. Auparavant, on aura examiné le "paradoxe du pouvoir " d'HIRSHLEIFER. Soit 2 joueurs en situation de conflit, qui ont chacun à choisir entre activités de production, de prédation ou diverses combinaisons des deux activités. Le modèle conclut à la préférence rationnelle du plus pauvre pour la prédation et du plus riche pour la production : le paradoxe aboutit, dans sa conclusion, à l'égalisation tendancielle des revenus (forme forte), au rapprochement de ces revenus (forme faible).

La question de la valeur du pouvoir destructif est moins évidente pour ce qui est de la fonction de production de règles, puisque le pouvoir destructif est, en ce cas, source de l'évaluation. On comprend dès lors que, pendant le déroulement de l'affrontement (guerre, révolution), la question de la souveraineté n'étant pas encore tranchée, il y ait des évaluations rivales et contradictoires. Ainsi, à propos de la première guerre du Golfe, M.VAHABI identifie 8 protagonistes avec des vecteurs d'évaluation plus ou moins divergents concernant la vie humaine tant du personnel militaire américain que de la population irakienne. A noter que pour l'un au moins de ces évaluateurs, la vie humaine n'a pas de prix et que pour tel autre, la vie de l'ennemi est dotée d'une valeur négative et donc, réciproquement, sa mort d'une valeur positive. On voit là, selon M.VAHABI, l'un des attributs de la souveraineté, celui de pouvoir déclarer la guerre.

De tout cela, on peut inférer une indétermination radicale des coûts et bénéfices pendant le déroulement de l'affrontement (guerre, révolution). Les coûts et bénéfices du conflit ne peuvent s'apprécier 
qu'ex post et collectivement, une fois stabilisés les nouveaux rapports de force. L'auteur soutient même que, pour ce qui est de la révolution, il faut un certain recul historique. Enfin, M.VAHABI conclut aux rendements croissants du pouvoir destructif dans sa fonction de production de règles et, a contrario, aux rendements décroissants du pouvoir destructif pour l'autre fonction.

Avec le chapitre 5, M.VAHABI aborde la question des sources du pouvoir destructif. Pour la traiter convenablement, il faut en revenir à la distinction fondamentale entre fonction d'appropriation et fonction de production de règles ou entre le pouvoir destructif comme moyen et le pouvoir destructif comme fin. On trouve alors deux sources spécifiques à la première fonction, la première étant la technologie de destruction, la seconde la commercialisation et l'industrialisation de cette technologie. On trouve également deux sources spécifiques à la deuxième fonction, à savoir la légitimité d'une part et, d'autre part, le consensus fondé sur une communauté politique et sur la relation entre les dominants et leurs alliés au sein de celle-ci. On trouve enfin deux sources communes aux deux fonctions, l'organisation et l'esprit d'entreprise.

S'en prenant au déterminisme économique qui donne fort logiquement la priorité au pouvoir constructif dans le développement historique, M.VAHABI souligne l'importance primordiale des révolutions et des innovations dans le domaine de la destruction et de l'art militaire. Si l'on ajoute l'extension de ces innovations par leur commercialisation et leur industrialisation, on peut en inférer une lecture historique très éclairante, en particulier sur les relations entre systèmes et pouvoirs politiques, systèmes et pouvoirs économiques et technologie de destruction. Et tout ceci vaut dans toutes les dimensions du politique : impérialismes et hiérarchie des puissances, formes locales du pouvoir, taille des entités politiques, mode de mobilisation et de contrôle des populations, etc. Ce qui vient d'être dit à propos du politique, vaut aussi dans toutes les dimensions de l'économique: domination ou non du marché, groupes sociaux contrôlant la propriété et le pouvoir économiques, modes de gouvernance, taille des unités économiques et formes de concurrence et enfin, relations entre pouvoirs économiques et pouvoirs politiques, etc. Bref, M.VAHABI renforce ici sa thèse : au commencement était le pouvoir destructif.

Il reste qu'une fois posées et imposées, souveraineté et règles doivent se maintenir et que la force brute n'y saurait suffire. La théorie du conflit rationnel échoue à expliquer la production de règles et la légitimité qui soutient ces règles, échoue à expliquer ce complexe d'adhésion identificatoire des dominés et de puissance de destruction des dominants qui maintient la cohésion d'une communauté. L'auteur souligne, in fine, l'importance de l'organisation et de l'esprit d'entreprise dans l'art du pouvoir 
destructif, qu'il s'agisse de guerre (NAPOLÉON), de crime (AL CAPONE) ou de révolution (LÉNINE), trois formes d'expression du pouvoir destructif comme création destructrice. Un des problèmes les plus importants à résoudre est de passer du free riding des périodes de transition ou de guerre à la coopération pour établir et stabiliser de nouvelles règles.

M.VAHABI conclut en constatant le développement technique énorme qui tend à donner le primat à la fonction d'appropriation au détriment de la fonction de production de règles. On en arrive alors à une tension paradoxale causée par ce développement entre l'appropriation liée à la première fonction d'une part et la cohésion sociale et la légitimité inhérentes à la fonction de production de règles d'autre part., tension qui pourrait bien caractériser l'époque actuelle.

Tout compte rendu est infidèle, tout résumé est un lit de Procuste. D'emblée, on s'excusera ici de ne pas avoir pu détailler et traduire les nombreux développements bien venus, bien argumentés, bien documentés. Il reste que le constat qui ouvre l'ouvrage avec la double référence au Kid de Charlie CHAPLIN et au "healthy highshooled graduate " de T.C.SCHELLING est fécond et que la réflexion de M.VAHABI appelle des suites et des explorations dans plusieurs directions. Il faut insister aussi sur la richesse de l'érudition, la pertinence des références, la finesse de l'argumentation et de la conceptualisation, surtout dans un champ très peu étudié et très peu exploité par les économistes, à l'exception notable, en langue française, de Christian SCHMIDT ${ }^{1}$, dont le titre de l'ouvrage fait écho à celui de Raymond $\mathrm{ARON}^{2}$. Sur la base de ce constat, l'auteur nous conduit à des analyses et conclusions dont certaines peuvent être comptés désormais comme autant d'acquis. Ainsi de la distinction, à propos du pouvoir destructif entre fonction d'appropriation ou de prédation et fonction de production de règles; en corollaire, l'idée que le pouvoir destructif est essentiel à toute institutionnalisation, toute sémantique sociale et que, par exemple, il n'est pas de bien privé ou collectif par nature : cette distinction s'opère au sein d'un ordre légitime à l'origine duquel il a toujours du pouvoir destructif. L’idée d'explorer du côté de la destruction s'est révélée féconde. Cette idée manifeste une ouverture intellectuelle peu commune chez les économistes. Un anthropologue, un sociologue, un politologue acquiesceraient volontiers à la démarche de l'auteur et ne seraient pas autrement choqués de l'importance accordée par celui-ci au pouvoir destructif. En particulier, on ne peut qu'approuver M.VAHABI

1 Schmidt Christian 1991, Penser la guerre, penser l'économie, Paris Odile Jacob

2 Aron Raymond 1976, Penser la guerre. Clausewitz, Paris Gallimard

148 Economie et Institutions $-n^{\circ} 8-1^{\text {er }}$ semestre 2006 
quand il met l'accent sur le rôle central de la technologie et de l'innovation militaires dans le développement historique.

En définitive, on retrouve ici une thématique récurrente voire lancinante sur le rôle de la force et de la violence dans l'histoire. Certes, cette thématique est quasiment absente de la tradition économique néo-classique. En revanche, et M.VAHABI a raison de le souligner, SMITH et RICARDO d'un côté, KEYNES de l'autre, abordent la question de la guerre, au moins sous l'angle de son financement. Quant à MARX, il installe le conflit radical et, dans une certaine mesure, la guerre et la violence au sein même de l'histoire et de la dynamique sociale : on y reviendra.

Mais il reste toutefois que l'objet premier de l'économiste n'est pas la destruction, la force, la guerre, la violence ou la révolution. Son objet premier serait plutôt, pour ce qui concerne la société moderne, toute processus social concourant à la couverture onéreuse et pacifique des besoins et désirs manifestés socialement, concourant à cette couverture par l'activité productive et son organisation, par l'échange libre entre agents consentants d'objets matériels ou immatériels et de droits de disposition, sur la base d'une référence commune à un médium monétaire et à des prix arrêtés conjointement, collectivement et librement, exprimés en cette unité monétaire. Max WEBER insiste à juste titre sur le caractère pacifique des procédures : "...le terme d"économie" dans son sens propre ne s'applique qu'à l'exercice pacifique d'un pouvoir de disposer de certaines prestations, et dont l'orientation première est économique " (souligné par M.W.) ${ }^{3}$. Ce n'est pas qu'il ignore l'importance de la force et de ce que M.VAHABI appelle le pouvoir destructif : "En ce qui concerne, maintenant, cette autre spécificité qu'a l'économie d'être pacifique, il faut voir qu'elle est primordiale ; s'il est vrai en effet que toute espèce de violence effective (rapine, guerre, révolution) est susceptible d'être orientée économiquement, elle répond néanmoins à des formes de légalités autres que le fait de subvenir à ses besoins par des moyens formellement pacifiques " (ibid.) La distinction weberienne entre activité économique ("...l'exercice pacifique d'un droit de disposition d'orientation essentiellement économique ") et activité à orientation économique (" toute activité qui [a] est orientée en principe en principe à d'autres fins mais qui tient compte dans son déroulement de "faits économiques"...ou qui $[b]$ est d'orientation essentiellement économique mais utilise pour parvenir à ses fins des moyens violents" [souligné par M.W.]) $)^{4}$ apparaît ici essentielle. Formellement, il n'y a pas d'incompatibilité avec le propos de

3 Weber Max 1923 - 1981 - [éd française 1991], Histoire économique, Paris Gallimard (p.8).

4 Weber Max 1921-1956-1967 - [éd française 1971], Economie et société, Paris Plon (p.62). 
M.VAHABI. En effet, Max WEBER reconnait que " toute forme d'activité même violente (par exemple belliqueuse) peut avoir une orientation économique". Mais, selon lui, il convient d'opposer "les moyens "économiques" aux moyens "politiques" " et de "distinguer entre le politique et 1"économique" " (ibid. p.63).

Pourquoi ce détour par Max WEBER ? C'est afin de localiser et de cerner, avec l'aide de la perspicacité de ce géant intellectuel trop peu travaillé par les économistes, la source d'une impression d'abord confuse et diffuse, puis d'un sentiment de plus en plus précis de désaccord de fond.

On notera d'abord le paradoxe d'un projet d'économie politique $\mathrm{du}$ pouvoir destructif fondé sur la critique et le refus de l'économisme. Corollairement, l'adversaire est ici l'increvable orthodoxie néo-classique, résumée dans la rationalité optimisatrice. Pourquoi une économie politique, au risque de tout embrouiller ? Paradoxe en effet, puisque le pouvoir destructif est, in fine, de l'ordre $\mathrm{du}$ politique au sens large et que le propos de l'auteur est bien de redonner la primauté au politique et à son fondement ultime, le pouvoir destructif ; de réaffirmer l'importance des facteurs politiques, idéologiques, institutionnels et l'importance de la force et du pouvoir de destruction dans le développement historique, y compris le développement économique. En passant, on relèvera que la réciproque de la destruction est ici la création, assimilée, hâtivement selon nous, à la production au sens économique. Pas étonnant que, en bon hétérodoxe, M.VAHABI pourfende en permanence la rationalité optimisatrice des néo-classiques. Seulement, notre auteur n'hésite pas, chemin faisant, à emprunter à l'adversaire honni, quelques outils de sa panoplie comme les coûts de transaction et, plus grave parce que plus typique de cette rationalité, le calcul à la marge du coût et de la satisfaction. Les emprunts sont, au demeurant, tout à fait pertinents et féconds, du moins du point de vue d'un lecteur critique. Le reproche est, certes, trop facile. Mais ce qui est ici posé c'est le statut des concepts, car, après tout, on peut ne pas adhérer à tel ou tel ensemble théorique ou analytique et se permettre d'emprunter quelques outils logiques ou conceptuels. Mais encore faut-il clarifier le contexte méthodologique voire épistémologique de cet emprunt !

Ces emprunts au raisonnement marginaliste, compréhensibles chez un institutionnaliste modérément hétérodoxe, sont d'autant plus frappants que le ton général de l'ouvrage est plutôt d'un marxisme plutôt...orthodoxe! On y décline sans réserve les concepts de valeur d'usage et de valeur d'échange et, dans les diverse formes et faces du pouvoir de destruction apparaît, en filigrane, la lutte de classes, cette violence fondamentale installée au cœur même du mouvement historique des sociétés. L'antiéconomisme affiché, la fascination pour la révolution comme 
rébellion, insurrection et coup de force accentuent l'impression première, laissant finalement transparaittre une version plutôt léniniste. Mais si l'échec de l'expérience soviétique est aujourd'hui tellement patent que, selon l'auteur même, elle n'est pas renouvelable, où veut nous emmener M.VAHABI ?

Cette finale en forme d'interrogation est une invite à prolonger la distinction autour d'un ouvrage original, ambitieux, argumenté, riche et faisant montre d'une culture historique trop rare chez les économistes pour ne pas être soulignée. 